Check for updates

Cite this: Mater. Chem. Front. 2020, 4, 965

Received 9th January 2020,

Accepted 29th January 2020

DOI: 10.1039/d0qm00010h

rsc.li/frontiers-materials

\title{
Aggregation enhances luminescence and photosensitization properties of a hexaiodo-BODIPY $\dagger$
}

\author{
P. P. Praveen Kumar, Pranjali Yadav, Asifkhan Shanavas (D) and \\ Prakash P. Neelakandan (D) *
}

\begin{abstract}
Intramolecular rotations are known to interfere with the excited-state processes resulting in luminescence quenching. Efforts aimed at restricting intramolecular rotations have led to the development of highly luminescent systems. Here we report the luminescence properties of a BODIPY molecule $\left(\mathbf{I}_{\mathbf{6}}\right)$ containing six iodo-substituents. The luminescence of $\mathbf{I}_{6}$ was highly quenched in the solution state whereas the conversion of this molecule into its nanoaggregates enhanced the emission intensity. Detailed photophysical studies and molecular simulations were employed to study the underlying mechanism which showed that the electron-withdrawing nature of iodine atoms and the peculiar structure of $I_{6}$ contributed towards the anomalous luminescence behaviour. Further, the luminescence and photosensitization properties of $\mathbf{I}_{6}$ were exploited for cell imaging and photodynamic therapy. Thus, our simple but intelligent molecular design yielded a multi-faceted molecule having excellent photophysical properties for potential photobiological applications.
\end{abstract}

\section{Introduction}

Molecular motions are critical in determining luminescence efficiency of molecules. Intramolecular rotations about a $\mathrm{C}-\mathrm{C}$ bond joining aromatic rings is a well-known non-radiative pathway in organic fluorophores. Any process that restricts the intramolecular rotations serves to hinder the non-radiative decays thus leading to enhanced emission. This principle served as the platform for the development of fluorophores exhibiting aggregation induced emission (AIE).$^{1-5}$ The signature of the AIE phenomenon is a weak emission in dilute solutions which intensifies upon aggregation or in the solid state. AIE is typically exhibited by molecules having propeller-type structures that allow intramolecular rotations in dilute solutions which facilitates non-radiative decays. Upon aggregation, the intramolecular rotations are restricted thereby hindering the non-radiative decay processes and eventually leading to an enhancement in emission. Because of the inverted emission characteristics, AIE luminogens were readily accepted in biology and materials science. These materials are ideal for biomedical applications because of the superior luminescence features and excellent stability in the aggregated state. ${ }^{6-11}$

Institute of Nano Science and Technology, Habitat Centre, Phase 10, (Sector 64), Mohali 160062, Punjab, India. E-mail: ppn@inst.ac.in

$\dagger$ Electronic supplementary information (ESI) available: Experimental details, characterization and spectroscopy data. See DOI: 10.1039/d0qm00010h
Boron-dipyrromethene (BODIPY) dyes are commonly used as fluorescent indicators, bio labelling agents, photosensitizers and as active material in optoelectronics owing to their exceptional optical and electronic properties. ${ }^{12-19}$ BODIPYs are known for their sharp absorption and emission bands, high fluorescence quantum yields and small Stokes shifts in dilute solutions. ${ }^{20}$ However, because of the hydrophobic core, nonfunctionalized BODIPYs undergo aggregation in the aqueous medium and their benchmark photophysical properties are compromised under these conditions. ${ }^{21}$ The utility of simple BODIPY molecules are thus limited for biological applications.

Efforts are ongoing to develop new BODIPYs that retain their excellent photophysical properties in solution as well as in the aggregated/solid state. ${ }^{22}$ A commonly adopted strategy in this direction is to attach bulky molecules on the main backbone to reduce the intramolecular rotations. ${ }^{23-32}$ In another approach, BODIPYs are substituted with known moieties that exhibit the AIE phenomenon. ${ }^{33-39}$ It has also been shown that molecules with twisted BODIPY cores show interesting luminescence properties. ${ }^{40-42}$ Although a fair amount of success has been achieved in terms of luminescence properties, many of these strategies required multi-step synthetic routes which limits their widespread utility. Moreover, functional groups should be judiciously selected so as to improve cellular uptake and reduce toxicity if these molecules are to be employed for biological applications. Thus, design and synthesis of simple BODIPY dyes that retain their excellent photophysical properties 
under a variety of conditions remains a challenging area of research.

Herein we report the synthesis and photophysical properties of a simple BODIPY molecule $\left(\mathbf{I}_{\mathbf{6}}\right)$ bearing six iodine atoms. Because of the peculiar structure, $\mathbf{I}_{6}$ exhibited low emission in solution which could be turned-on by aggregation. Iodine atoms facilitated intersystem crossing thus allowing generation of singlet oxygen. Preliminary photobiological studies revealed that this molecule has the potential to be used as an efficient photosensitizer for photodynamic therapy (PDT).

\section{Results and discussion}

The synthesis of the BODIPY molecule $\mathbf{I}_{\mathbf{6}}$ was achieved as shown in Fig. 1a. 1,3,5-Tribenzene carbonyl chloride on condensation with 2,4-dimethylpyrrole followed by complexation with $\mathrm{BF}_{3} \cdot \mathrm{Et}_{2} \mathrm{O}$ yielded the BODIPY $\mathbf{B}_{3}$ in moderate yields. ${ }^{43}$ Iodination of $\mathbf{B}_{3}$ with $\mathrm{N}$-iodosuccinimide in dichloromethane gave $\mathbf{I}_{6}$ in $88 \%$ yield. Both $\mathbf{B}_{3}$ and $\mathbf{I}_{6}$ were characterized by NMR and high resolution mass spectrometry (Fig. S1, ESI $\dagger$ ).

At the outset, we were interested in studying the photophysical properties of $\mathbf{B}_{3}$ and $\mathbf{I}_{\mathbf{6}}$ under a variety of conditions. As shown in Fig. 2 and Table $1, \mathbf{B}_{3}$ and $\mathbf{I}_{\mathbf{6}}$ showed absorption maxima at 502 and $520 \mathrm{~nm}$, respectively in acetonitrile whereas the corresponding fluorescence maxima were observed at 525 and $550 \mathrm{~nm}$. $\mathbf{B}_{3}$ was observed to be negligibly fluorescent in solution $\left(\phi_{\mathrm{f}}=0.0005^{43}\right)$ whereas $\mathbf{I}_{\mathbf{6}}$ was observed to be moderately fluorescent. The fluorescence quantum yield and lifetime of $\mathbf{I}_{6}$ were found to be 0.03 and $1.65 \mathrm{~ns}$ in acetonitrile (Fig. S2, ESI $\dagger)$, and the radiative $\left(k_{\mathrm{r}}\right)$ and non-radiative $\left(k_{\mathrm{nr}}\right)$ decay constants were calculated to be $1.8 \times 10^{8}$ and $1.8 \times 10^{7} \mathrm{~s}^{-1}$, respectively. It is noteworthy that $\mathbf{I}_{\mathbf{6}}$ was observed to be fluorescent in the solid state as well. The solid state absorption
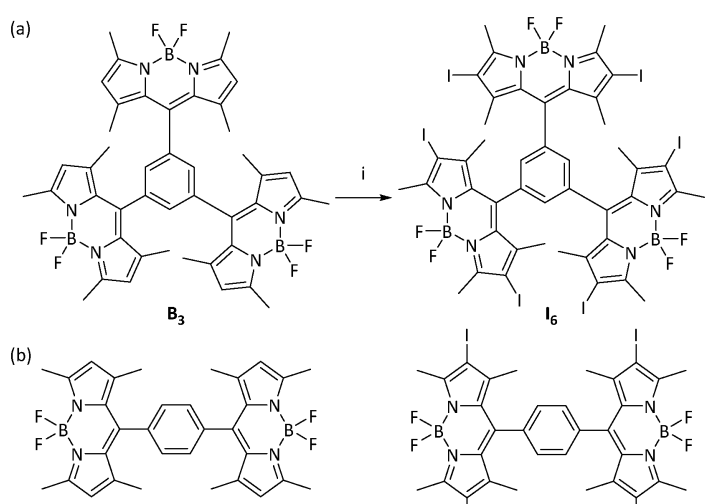

$\mathrm{B}_{2}$
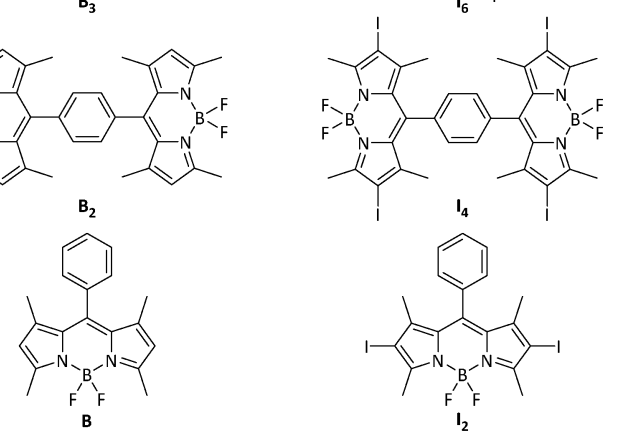

Fig. 1 (a) Synthesis of $\mathbf{I}_{\mathbf{6}}$. Reactions conditions. (i) $\mathrm{N}$-lodosuccinimide, dichloromethane, $25^{\circ} \mathrm{C}, 4 \mathrm{~h}$. (b) Chemical structures of related BODIPY molecules reported elsewhere. ${ }^{44,45}$

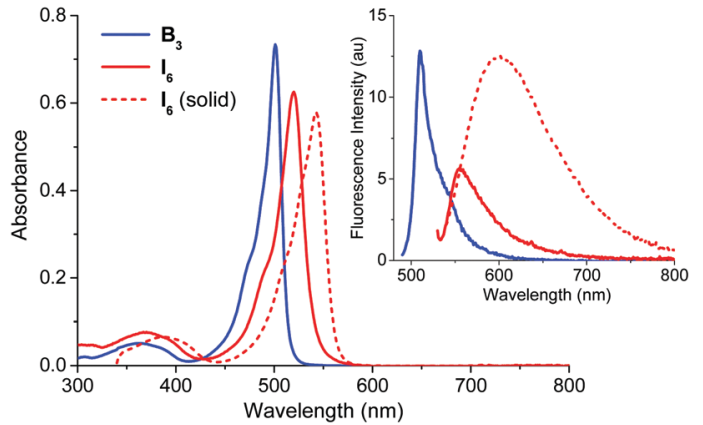

Fig. 2 Absorption and (inset) emission spectra of $\mathbf{B}_{\mathbf{3}}(6 \mu \mathrm{M})$ and $\mathbf{I}_{\mathbf{6}}(4.5 \mu \mathrm{M})$ in acetonitrile (solid lines) and that of $\mathbf{I}_{\mathbf{6}}$ in the solid state (dashed lines). The emission spectra were obtained by exciting the samples at the respective absorption maxima.

Table 1 Photophysical properties of various BODIPY molecules

\begin{tabular}{|c|c|c|c|c|c|c|c|c|}
\hline & \multicolumn{2}{|c|}{$\lambda_{\mathrm{abs}}(\mathrm{nm})$} & \multicolumn{2}{|c|}{$\lambda_{\mathrm{em}}(\mathrm{nm})$} & \multicolumn{2}{|l|}{$\phi_{\mathrm{f}}$} & \multicolumn{2}{|l|}{$\underline{\phi_{\Delta}}$} \\
\hline & $a$ & $b$ & $a$ & $b$ & $a$ & $b$ & $a$ & $b$ \\
\hline B & $501^{45}$ & $550^{46}$ & $510^{45}$ & $505^{46}$ & $0.64^{45}$ & $c$ & $0.008^{46}$ & $c$ \\
\hline $\mathbf{B}_{2}$ & 502 & & & & $0.02^{47}$ & $c$ & 0.071 & $c$ \\
\hline $\mathbf{B}_{3}$ & 501 & 502 & 510 & 550 & $0.0005^{43}$ & 0.013 & & $d$ \\
\hline $\mathrm{I}_{2}$ & $533^{45}$ & $c$ & $537^{45}$ & $c$ & $0.02^{45}$ & & $0.73^{45}$ & $c$ \\
\hline $\mathbf{I}_{4}$ & $540^{44}$ & $c$ & $c$ & $c$ & $c$ & $c$ & $0.68^{44}$ & $c$ \\
\hline $\mathbf{I}_{6}$ & 520 & 506 & 550 & 566 & 0.03 & 0.15 & 0.21 & 0.82 \\
\hline
\end{tabular}

${ }^{a}$ In solution. ${ }^{b}$ As aggregates in aqueous medium. ${ }^{c}$ Not reported. ${ }^{d}$ Not determined as photo-bleaching was observed.

spectrum of $\mathbf{I}_{\mathbf{6}}$ was slightly broader with a maximum at $542 \mathrm{~nm}$ and the emission maximum appeared at $604 \mathrm{~nm}$. The fluorescence lifetime of $\mathbf{I}_{\mathbf{6}}$ was significantly longer ( $\tau=4.71 \mathrm{~ns}$ ) in the solid state as compared to that in solution. On the other hand, $\mathbf{B}_{3}$ was not observed to be emissive in the solid state. The bathochromically shifted absorption and emission peaks and longer fluorescence lifetime of $\mathbf{I}_{\mathbf{6}}$ in the solid state indicates aggregation of the molecules in the solid state.

The absorption and emission properties of $\mathbf{B}_{\mathbf{3}}$ and $\mathbf{I}_{\mathbf{6}}$ are anomalous as compared to typical BODIPY dyes which are known for their sharp absorption and fluorescence spectra with high quantum yields that are typically independent of their environment. To gain insights into the luminescence behaviour of $\mathbf{I}_{6}$, we studied the effect of temperature and viscosity on the fluorescence of $\mathbf{I}_{\mathbf{6}}$. When a solution of $\mathbf{I}_{\mathbf{6}}$ in acetonitrile was heated from 5 to $60{ }^{\circ} \mathrm{C}$, the absorption spectrum remained unaffected whereas the emission intensity decreased regularly (Fig. 3a). At $60{ }^{\circ} \mathrm{C}$, the fluorescence emission intensity was $\sim 6$ times lower as compared to that at $5{ }^{\circ} \mathrm{C}$. The effect of viscosity on the absorption and emission spectra of $\mathbf{I}_{\mathbf{6}}$ was then studied. When a highly viscous solvent such as glycerol was added to a solution of $\mathbf{I}_{\mathbf{6}}$ in acetonitrile, we observed broadening of the absorption spectrum (Fig. 3b). Further, a new peak emerged at $463 \mathrm{~nm}$ and became prominent as the amount of glycerol was increased to $90 \%$. Under similar conditions, the fluorescence intensity of $\mathbf{I}_{\mathbf{6}}$ was observed to increase $\sim 17$ times. However, we observed turbidity in the solution as the amount of glycerol was increased above $50 \%$ thereby indicating the aggregation of $\mathbf{I}_{\mathbf{6}}$. 

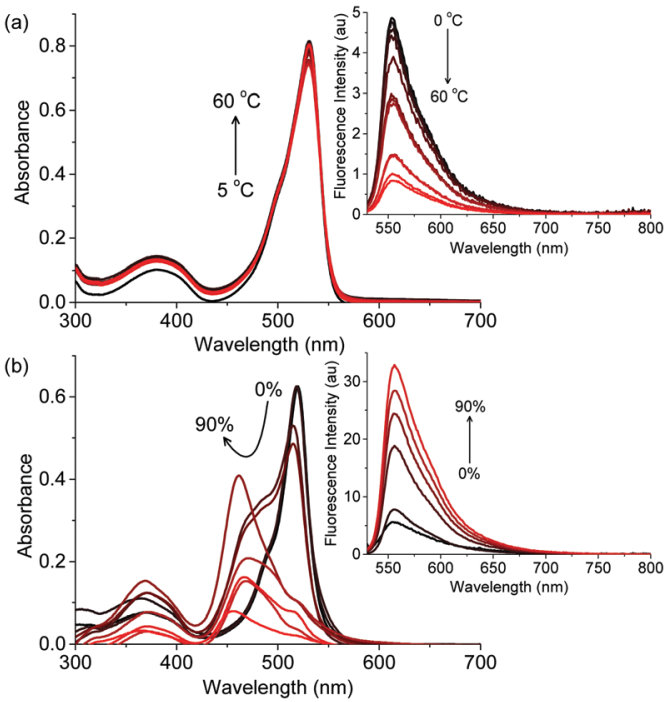

Fig. 3 Changes in the absorption and (inset) emission spectra of $\mathbf{I}_{6}$ $(4.5 \mu \mathrm{M})$ as a function of varying (a) temperature and (b) amount of glycerol in acetonitrile. $\lambda_{\mathrm{ex}}$ is $520 \mathrm{~nm}$.

As glycerol was promoting aggregation of $\mathbf{I}_{\mathbf{6}}$, we were intrigued to investigate whether other solvents induced similar changes. In this context, the effect of solvent polarity was evaluated by recording the absorption and emission of $\mathbf{I}_{\mathbf{6}}$ in different solvents. We observed that in non-polar solvents $\mathbf{I}_{\mathbf{6}}$ showed a red-shift of $\sim 20 \mathrm{~nm}$ in the absorption maximum as compared to that in polar solvents (Fig. S3 and S4, ESI $\dagger$ ). A similar trend was observed in the emission behaviour as well wherein we observed a maximum at 545-550 nm in polar solvents whereas in non-polar solvents the emission maximum was red shifted by $\sim 40 \mathrm{~nm}$. These observations indicated the non-polar character of the $\mathbf{I}_{\mathbf{6}}$ molecule.

Our next objective was to study the aggregation of $\mathbf{I}_{\mathbf{6}}$ in acetonitrile-water mixtures. $\mathbf{I}_{6}$, which showed an absorption peak at $520 \mathrm{~nm}$ in acetonitrile, exhibited significantly broadened and red-shifted absorption upon increasing water content. In $90 \%$ water-acetonitrile mixture, the long-wavelength absorption band was observed to redshift by $7 \mathrm{~nm}$ and a new sharp absorption peak appeared at $445 \mathrm{~nm}$ (Fig. 4a). The fluorescence intensity of $\mathbf{I}_{\mathbf{6}}$ was observed to increase with the addition of water and we noticed 13-times enhancement in the fluorescence in $90 \%$ wateracetonitrile mixture. The fluorescence quantum yield of $\mathbf{I}_{\mathbf{6}}$ determined in $90 \%$ water-acetonitrile mixture was found to be $\phi_{\mathrm{f}}=0.075$ which was 4-times higher than that in acetonitrile. Moreover, these changes were significant enough to result in visual changes in the fluorescence of $\mathbf{I}_{\mathbf{6}}$ and we observed a change in colour of $\mathbf{I}_{\mathbf{6}}$ from transparent pale green in acetonitrile to bluishgreen in $90 \%$ water-acetonitrile mixture. Under similar experimental conditions, we also studied the effect of aggregation on the photophysical properties of $\mathbf{B}_{\mathbf{3}}$. However, in contrast to the results obtained with $\mathbf{I}_{\mathbf{6}}, \mathbf{B}_{\mathbf{3}}$ exhibited negligible changes in the absorption and emission spectra in acetonitrile-water mixtures thereby ruling out aggregation induced emission (Fig. S5, ESI $\dagger$ ).

Since heavy atoms like bromine and iodine are known to enhance the intersystem crossing efficiency of organic dyes, ${ }^{48-51}$
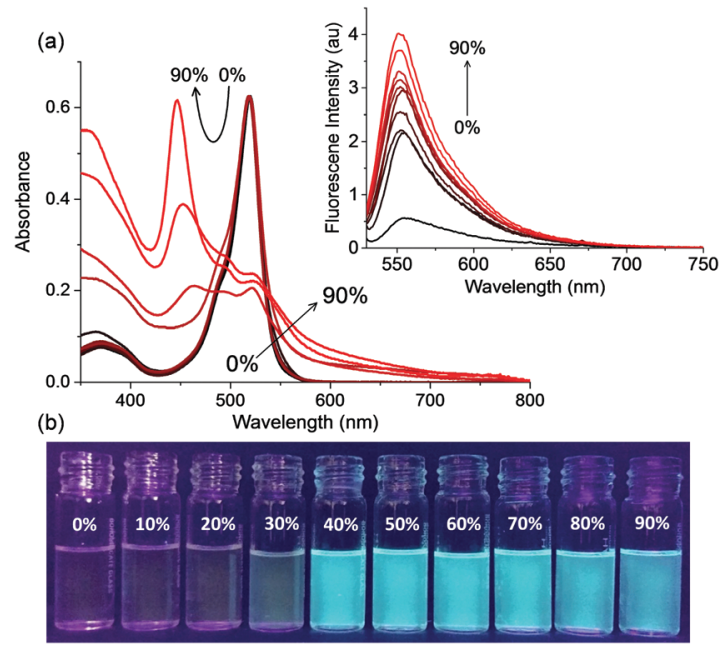

Fig. 4 (a) Absorption and (inset) emission spectra of $I_{6}(4.5 \mu M)$ in acetonitrile-water mixtures. $\lambda_{\mathrm{ex}}=520 \mathrm{~nm}$. (b) Visual fluorescence of $\mathbf{I}_{6}$ upon excitation with a UV lamp $\left(\lambda_{\mathrm{ex}}=365 \mathrm{~nm}\right)$ in acetonitrile-water mixtures.

we inferred that triplet excited states will be formed in $\mathbf{I}_{\mathbf{6}}$. The formation of triplet excited states can facilitate generation of reactive oxygen species thus making $\mathbf{I}_{\mathbf{6}}$ suitable as a photosensitizer for photodynamic therapy. To test this hypothesis, we studied the photosensitization properties of $\mathbf{I}_{\mathbf{6}}$ in acetonitrile using 1,3-diphenylisobenzofuran (DPBF) as a trapping agent for singlet oxygen. On photoirradiation of a mixture of $\mathbf{I}_{\mathbf{6}}$ and DPBF in acetonitrile, we observed a gradual decrease in the absorbance of DPBF at $420 \mathrm{~nm}$ whereas the absorbance of $\mathbf{I}_{\mathbf{6}}$ at $520 \mathrm{~nm}$ remained unperturbed (Fig. S6 and S7, ESI $\dagger$ ). These results indicate that $\mathbf{I}_{\mathbf{6}}$ was photostable under our experimental conditions and that it sensitizes the generation of singlet oxygen. The presence of singlet oxygen was further confirmed by recording its characteristic emission at $1290 \mathrm{~nm}^{52}$ in acetonitrile (Fig. S8, ESI $\dagger$ ). The efficiency of singlet oxygen generation of $\mathbf{I}_{\mathbf{6}}$ was quantified by calculating the quantum yield using methylene blue as a reference standard and was obtained as $\phi_{\Delta}=0.21$ (Fig. S9, ESI $\dagger$ ).

As $\mathbf{I}_{\mathbf{6}}$ exhibited enhanced emission in acetonitrile-water mixtures and was capable of generating singlet oxygen, we were interested in studying if nanoaggregates of $\mathbf{I}_{\mathbf{6}}$ could be synthesized for studying the photosensitization properties in the aqueous medium. These nanoaggregates would be dispersible in the aqueous medium and would show superior fluorescence and photosensitization properties thereby making it an ideal active ingredient for image-guided PDT. Nanoaggregates of $\mathbf{I}_{\mathbf{6}}$ (denoted hereafter as $\mathbf{I}_{\mathbf{6}}$-NA) were synthesized by the re-precipitation method wherein a concentrated solution of $\mathbf{I}_{\mathbf{6}}$ in acetone was added to large excess of water and the nanoaggregates were collected by centrifugation (see the experimental section for the detailed procedure). $\mathbf{I}_{\mathbf{6}}$-NA was dispersed in water and characterized by dynamic light scattering (DLS) and microscopy. As shown in Fig. S10 (ESI $\dagger$ ), DLS analysis showed an average particle size of $85.6 \pm 3.1 \mathrm{~nm}$ for $\mathbf{I}_{\mathbf{6}}$-NA. Similarly, high resolution transmission electron microscopy measurements (Fig. 5a) showed the presence of spherical particles and the 


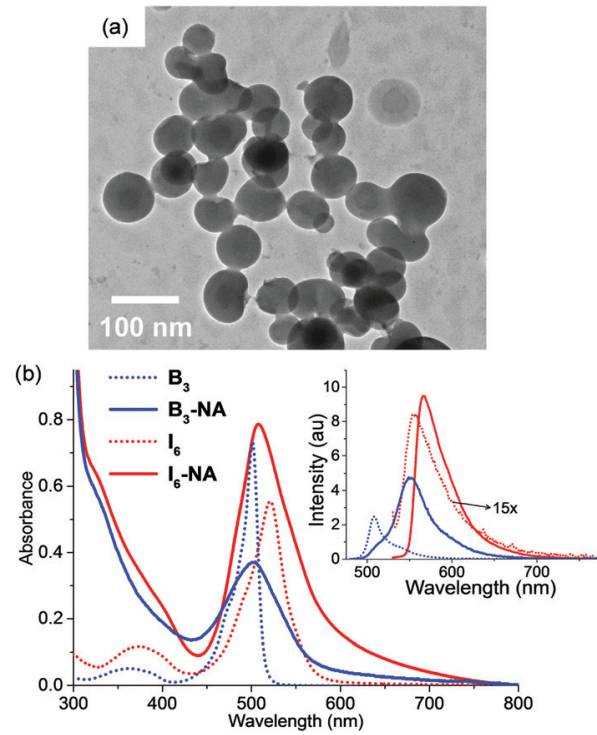

Fig. 5 (a) Transmission electron micrograph of $\mathbf{I}_{\mathbf{6}}$-NA in water (b) Absorption and (inset) fluorescence spectra of $\mathbf{B}_{\mathbf{3}}(6 \mu \mathrm{M})$ and $\mathbf{I}_{\mathbf{6}}(4.5 \mu \mathrm{M})$ in acetonitrile and $\mathbf{B}_{3}-\mathbf{N A}\left(10 \mu \mathrm{g} \mathrm{mL}^{-1}\right)$ and $\mathbf{I}_{\mathbf{6}}-\mathbf{N A}\left(10 \mu \mathrm{g} \mathrm{mL}^{-1}\right)$ in water. The emission spectra were obtained by exciting the samples at the respective absorption maxima.

particle size corroborated well with that obtained from DLS experiments.

It is assumed that $\mathbf{I}_{\mathbf{6}}$ undergoes spontaneous self-assembly during the nanoaggregate formation the evidence for which was obtained from ${ }^{19} \mathrm{~F}$ NMR spectroscopy. As shown in Fig. S11 (ESI $\dagger$ ), $\mathbf{I}_{6}$ in $\mathrm{CDCl}_{3}$ showed a multiplet from $\delta-145.94$ to -146.28 ppm corresponding to the $-\mathrm{BF}_{2}$ moieties. On the other hand, we observed a broad singlet at $-119.28 \mathrm{ppm}$ in the ${ }^{19} \mathrm{~F}$ NMR spectrum of $\mathbf{I}_{6}-\mathbf{N A}$ in $\mathrm{D}_{2} \mathrm{O}$. Although a comparison of the NMR spectra in organic and aqueous media are inappropriate, the peak broadening and the downfield shift indicates the aggregation of $\mathbf{I}_{\mathbf{6}}$ in $\mathbf{I}_{\mathbf{6}}$-NA. The formation of $\mathbf{I}_{\mathbf{6}}$-NA was also accompanied by changes in the absorption and emission spectra of $\mathbf{I}_{\mathbf{6}}$ (Fig. $5 \mathrm{~b}$ ). $\mathbf{I}_{\mathbf{6}} \mathbf{- N A}$ showed a broad absorption peak that was blue shifted by $13 \mathrm{~nm}$ as compared to $\mathbf{I}_{\mathbf{6}}$ in acetonitrile whereas the emission maximum for $\mathbf{I}_{\mathbf{6}}$-NA was observed at $568 \mathrm{~nm}$. The fluorescence quantum yield and lifetime of $\mathbf{I}_{6}-\mathbf{N A}$ were observed to be 0.15 and $3.12 \mathrm{~ns}$, respectively in water which were substantially higher as compared to that of $\mathbf{I}_{\mathbf{6}}$ in acetonitrile. Similarly, the radiative $\left(k_{\mathrm{r}}=4.8 \times 10^{8} \mathrm{~s}^{-1}\right)$ and non-radiative $\left(k_{\mathrm{nr}}=4.8 \times 10^{7} \mathrm{~s}^{-1}\right)$ decay constants were also observed to be higher in the aggregated state as compared to that in acetonitrile. Nanoaggregates of $\mathbf{B}_{3}$ (denoted as $\mathbf{B}_{3}$-NA) were also synthesized by following a similar procedure. Fig. S10 (ESI $\dagger$ ) and Fig. 5b shows the characterization data and the absorption and emission spectra of $\mathbf{B}_{3}$-NA. It is remarkable to note that $\mathbf{B}_{3}$-NA was observed to be negligibly fluorescent with a quantum yield of 0.013 and this observation was in stark contrast to the fluorescence properties of $\mathbf{I}_{\mathbf{6}}$-NA.

Next, we were interested in studying the photosensitization properties of the nanoaggregates in water. Similar to the experiments carried out in acetonitrile, the singlet oxygen generation

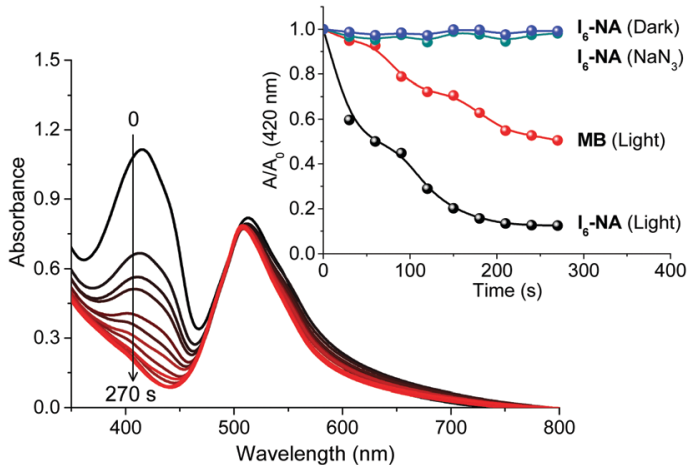

Fig. 6 Changes in the UV-Vis absorption spectrum of a mixture of 1,3-diphenylisobenzo-furan (DPBF, $90 \mu \mathrm{M})$ and $\mathrm{I}_{6}$-NA $\left(10 \mu \mathrm{g} \mathrm{mL} \mathrm{m}^{-1}\right)$ as a function of irradiation time in $20 \%$ ethanol-water. Inset shows the relative decrease in the absorption of DPBF at $420 \mathrm{~nm}$ in the presence of $\mathbf{I}_{\mathbf{6}}-\mathbf{N A}$ or methylene blue (MB) under different experimental conditions.

ability of $\mathbf{I}_{\mathbf{6}}$-NA was studied using DPBF as a singlet oxygen trap. As shown in Fig. 6, we observed a gradual decrease in the absorbance of DPBF at $410 \mathrm{~nm}$ upon photoirradiating a mixture of $\mathbf{I}_{\mathbf{6}}$-NA and DPBF. It is noteworthy that $\mathbf{I}_{\mathbf{6}}$-NA was highly efficient in generating singlet oxygen as compared to $\mathbf{I}_{\mathbf{6}}$ and the quantum yield of singlet oxygen generation was found to be 0.82 (Fig. S9, ESI $\dagger$ ). The formation of singlet oxygen was further confirmed by monitoring the emission of ${ }^{1} \mathrm{O}_{2}$ at $1280 \mathrm{~nm}$ in water (Fig. S8, ESI $\dagger$ ). Further, control experiments carried out in dark and in the presence of sodium azide conclusively proved the photosensitized generation of singlet oxygen by $\mathbf{I}_{\mathbf{6}}$-NA.

Our results indicate that $\mathbf{I}_{\mathbf{6}}$-NA generates singlet oxygen efficiently in the aqueous medium upon photoirradiation. The efficacy of $\mathbf{I}_{\mathbf{6}} \mathbf{-} \mathbf{- N A}$ as photodynamic agents was tested against C6-glioblastoma cell line upon photoirradiation. When the cells were incubated with different concentrations of $\mathbf{I}_{6}$-NA such as 5 , 10 and $20 \mu \mathrm{g} \mathrm{mL} \mathrm{m}^{-1}$ and photoirradiated for 10 minutes, we observed up to $\sim 60 \%$ cell death (Fig. $7 \mathrm{a}$ ). On the other hand, cells incubated with similar concentrations of $\mathbf{I}_{\mathbf{6}}$-NA and kept in dark conditions were observed to be almost $100 \%$ viable thereby confirming photosensitized generation of singlet oxygen only in light conditions. Since the nanoaggregates were fluorescent, their cellular localization was studied by incubating $\mathbf{I}_{\mathbf{6}}$-NA with C6 cells followed by fixation and treatment with DAPI/ Phalloidin-TRITC (nuclear/cytoskeletal labels) prior to imaging by confocal microscopy. As $\mathbf{I}_{\mathbf{6}}$-NA exhibited an emission that tailed up to $\sim 750 \mathrm{~nm}$, we could image the cells in the green and red channels. It was observed that $\mathbf{I}_{6}$-NA localized in the cytoplasm around the nuclear region when simultaneously scanned with 405/488/561 nm excitations. This was exemplified in the merged image of Fig. $7 \mathrm{~b}$ wherein the $\mathbf{I}_{\mathbf{6}}$-NA appears yellow around the blue region. Phalloidin-TRITC assisted in marking the boundary of the cells and its emission was clearly limited only to the cell surface as seen in control.

BODIPYs are known to be highly emissive in solution with many molecules exhibiting fluorescence quantum yield close to unity. ${ }^{16,20,53-55}$ However, $\mathbf{B}_{3}$ is an exception and it was observed to be negligibly fluorescent. The peculiar structure of $\mathbf{B}_{3}$ could 
(a)

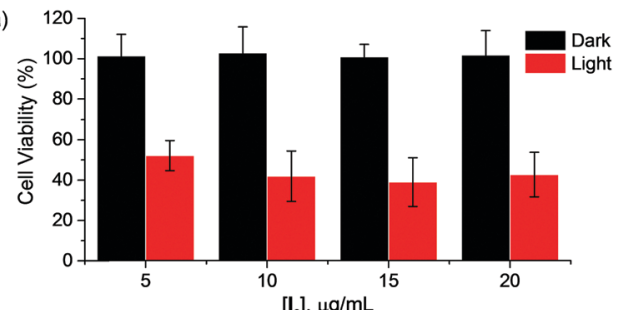

(b)

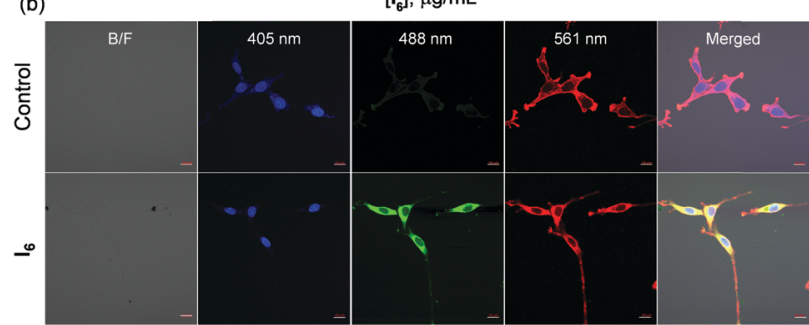

Fig. 7 (a) In vitro phototoxicity in $\mathrm{C} 6$ cells treated with $\mathrm{I}_{6}$-NA under dark and light conditions. (b) Confocal microscopic image of $\mathrm{C} 6$ cells treated with $\mathbf{I}_{6}$-NA $\left(12.5 \mu \mathrm{g} \mathrm{mL}^{-1}\right)$ excited at different wavelengths. The cells were stained with nuclear staining dye Hoechst 33342 and phalloidin-TRITC. Scale bar is $10 \mu \mathrm{m}$.

have contributed to its non-emissive character. Molecular simulations indicate that the BODIPY moieties are oriented orthogonally as compared to the central phenyl ring (Fig. 8). As compared to the previously reported monomeric and dimeric BODIPYs $\mathbf{B}$ and $\mathbf{B}_{\mathbf{2}}$ (Fig. 1b), BODIPY cores in $\mathbf{B}_{\mathbf{3}}$ are arranged in close proximity with an average distance of $7.649 \AA$ between the BODIPY units. It is known that the presence of multiple chromophores in close proximity often leads to exciton coupling between dyes thereby resulting in significant changes in the absorption and emission properties. ${ }^{56-59}$ Thus, the presence of three BODIPY units around the central phenyl ring plausibly promotes intramolecular interactions thereby resulting in quenching of fluorescence. The observation of negligible difference in the absorbance but a significant red-shift in the emission maximum of $\mathbf{B}_{\mathbf{3}}$ as compared to $\mathbf{B}$ and $\mathbf{B}_{\mathbf{2}}$ (Table 1) suggests excited state interactions. Further, intramolecular rotations about the $\mathrm{C}-\mathrm{C}$ bond joining

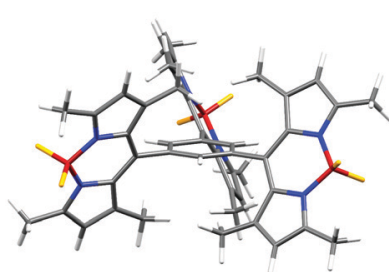

$\mathbf{B}_{3}$

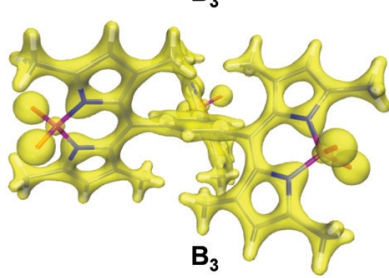

Fig. 8 Optimized geometry of $\mathbf{B}_{\mathbf{3}}$ and $\mathbf{I}_{\mathbf{6}}$ along with their electron cloud distribution. the BODIPY unit to the phenyl ring contribute towards the quenching effect.

The photophysical behaviour of $\mathbf{I}_{\mathbf{6}}$ in acetonitrile was on expected lines and was comparable to $\mathbf{I}_{2}$ and $\mathbf{I}_{\mathbf{4}}$. It was recently reported that $\mathbf{I}_{\mathbf{4}}$ having four iodine atoms fared poorly against $\mathbf{I}_{\mathbf{2}}$ having two iodines in terms of singlet oxygen generation. ${ }^{44}$ This observation was rationalized on the basis of the competition between heavy atom effect and configuration of $\mathbf{I}_{\mathbf{4}}$. Our results support this observation wherein $\mathbf{I}_{\mathbf{6}}$ exhibited a further lower singlet oxygen generation efficiency of 0.21 in acetonitrile as compared to 0.73 and 0.68 for $\mathbf{I}_{2}$ and $\mathbf{I}_{4}$, respectively. However, we observed a dramatic change in the photophysical behaviour of $\mathbf{I}_{\mathbf{6}}$ upon aggregation: the fluorescence was turned-on and a substantial increase was observed in the generation of singlet oxygen. We propose a model for the aggregation of $\mathbf{I}_{\mathbf{6}}$ as depicted in Fig. 9 for explaining the unforeseen behaviour of the aggregates of $\mathbf{I}_{\mathbf{6}}$. Because of the peculiar geometry consisting of the orthogonal BODIPY units, the chromophore (BODIPY) in the $\mathbf{I}_{\mathbf{6}}$ molecule cannot form aggregates in a conventional manner wherein a large number of the chromophores self-assemble through non-covalent interactions (Fig. 9a). Instead, the selfassembly is limited to the formation of $\mathrm{H}$-type dimers with respect to the chromophore units. The observation of blue-shifted absorption in glycerol and in acetonitrile-water mixture exemplifies this proposition. However, the aggregation restricts the rotation of the BODIPY units and enhances the emission intensity and the efficiency of singlet oxygen generation. ${ }^{60}$ It may also be noted that the increased absorption cross-section of $\mathbf{I}_{\mathbf{6}}$-NA in water as compared to $\mathbf{I}_{\mathbf{6}}$ in acetonitrile also contributes to the enhanced ${ }^{1} \mathrm{O}_{2}$ generation efficiency.

(a)

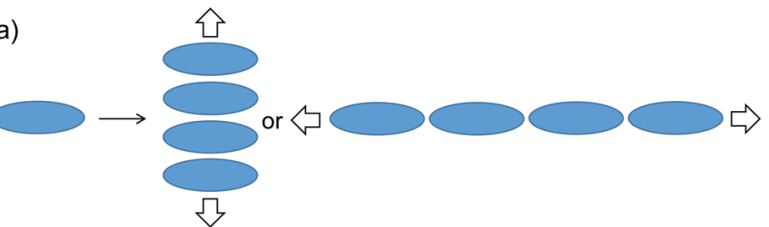

(b)

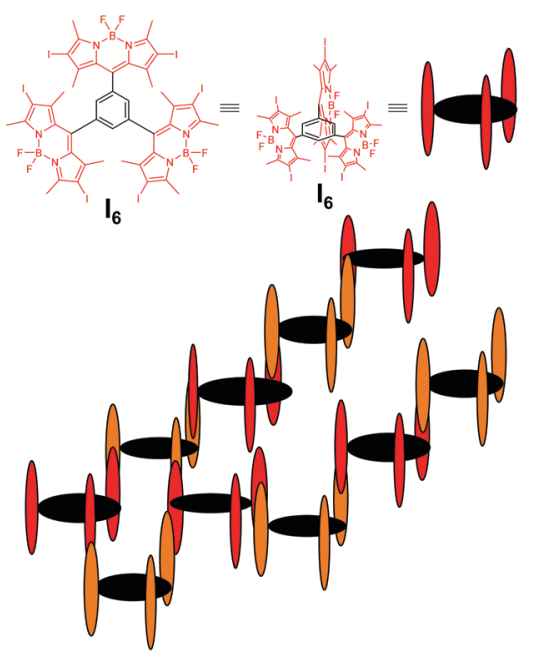

Fig. 9 Schematic representation of aggregation in (a) conventional planar molecules and (b) $\mathbf{I}_{6}$. The alternate molecules of $\mathbf{I}_{6}$ are represented in different colours for better visualization. 
It is intriguing to note that despite having similar structures, the aggregates of $\mathbf{B}_{\mathbf{3}}$ and $\mathbf{I}_{\mathbf{6}}$ exhibited extremely different fluorescence properties. The key to the remarkable photophysical properties of $\mathbf{I}_{\mathbf{6}}$-NA is the presence of the iodo-substituents. It is proposed that the iodo-groups force the BODIPY units to stack in an off-set manner because of their steric bulkiness. Moreover, iodine atoms are known to form halogen bonds ${ }^{61}$ and thus would promote intermolecular interactions. This in turn would reduce the intramolecular excitonic interactions between the individual BODIPY units in $\mathbf{I}_{\mathbf{6}}$ as compared to that in $\mathbf{B}_{\mathbf{3}}$ thereby resulting in an enhanced fluorescence and photosensitization efficiency. The progressive red-shift in the emission spectrum of $\mathbf{I}_{\mathbf{6}}$ from solution to the aggregated state in water and further to the solid state could also be rationalized on the basis of halogen bonding which would be stronger in the solid state as compared to that in $\mathbf{I}_{\mathbf{6}}$-NA in water due to the competition from water molecules.

\section{Conclusions}

We synthesised a hexaiodinated BODIPY molecule with interesting photophysical properties. The molecule showed low fluorescence in acetonitrile whereas the emission was enhanced significantly in the aggregated state. Similarly, the photosensitization properties were also observed to be more efficient in the aggregated state as compared to that in solution. Photobiological studies showed that this molecule was capable of functioning as a photosensitizer and imaging agent for photodynamic therapy. Molecular simulations indicated that the peculiar arrangement of the BODIPY units and the presence of iodine atoms as responsible for its emission characteristics.

\section{Conflicts of interest}

There are no conflicts to declare.

\section{Acknowledgements}

This work was supported by the Science and Engineering Research Board, New Delhi (PDF/2017/001221). We thank Mr Aritra Mukhopadhyaya (INST) and Dr Md. Ehesan Ali (INST) for molecular simulations, Prof. Sameer Sapra, Department of Chemistry, IIT Delhi for help with luminescence measurements and SAIF (Panjab University, Chandigarh) for analytical facilities.

\section{References}

1 Y. Chen, J. W. Y. Lam, R. T. K. Kwok, B. Liu and B. Z. Tang, Aggregation-Induced Emission: Fundamental Understanding and Future Developments, Mater. Horiz., 2019, 6, 428-433.

2 Y. Li, S. Liu, T. Han, H. Zhang, C. Chuah, R. T. K. Kwok, J. W. Y. Lam and B. Z. Tang, Sparks Fly When AIE Meets with Polymers, Mater. Chem. Front., 2019, 3, 2207-2220.

3 Z. He, C. Ke and B. Z. Tang, Journey of Aggregation-Induced Emission Research, ACS Omega, 2018, 3, 3267-3277.
4 H. Wang, E. Zhao, J. W. Y. Lam and B. Z. Tang, AIE Luminogens: Emission Brightened by Aggregation, Mater. Today, 2015, 18, 365-377.

5 Y. Hong, J. W. Y. Lam and B. Z. Tang, Aggregation-Induced Emission, Chem. Soc. Rev., 2011, 40, 5361-5388.

6 H.-B. Cheng, Y. Li, B. Z. Tang and J. Yoon, Assembly Strategies of Organic-Based Imaging Agents for Fluorescence and Photoacoustic Bioimaging Applications, Chem. Soc. Rev., 2020, 49, 21-31.

7 K. C. Chong, F. Hu and B. Liu, AIEgen Bioconjugates for Specific Detection of Disease-Related Protein Biomarkers, Mater. Chem. Front., 2019, 3, 12-24.

$8 \mathrm{H}$. Wang and G. Liu, Advances in Luminescent Materials with Aggregation-Induced Emission (AIE) Properties for Biomedical Applications, J. Mater. Chem. B, 2018, 6, 4029-4042.

9 H. Gao, X. Zhao and S. Chen, AIEgen-Based Fluorescent Nanomaterials: Fabrication and Biological Applications, Molecules, 2018, 23, 419.

10 J. Mei, Y. Huang and H. Tian, Progress and Trends in AIE-Based Bioprobes: A Brief Overview, ACS Appl. Mater. Interfaces, 2018, 10, 12217-12261.

11 J. Qian and B. Z. Tang, AIE Luminogens for Bioimaging and Theranostics: From Organelles to Animals, Chem, 2017, 3, 56-91.

12 M. A. Filatov, Heavy-Atom-Free BODIPY Photosensitizers with Intersystem Crossing Mediated by Intramolecular Photoinduced Electron Transfer, Org. Biomol. Chem., 2020, 18, 10-27.

13 A. Turksoy, D. Yildiz and E. U. Akkaya, Photosensitization and Controlled Photosensitization with BODIPY Dyes, Coord. Chem. Rev., 2019, 379, 47-64.

14 P. Kaur and K. Singh, Recent Advances in the Application of BODIPY in Bioimaging and Chemosensing, J. Mater. Chem. C, 2019, 7, 11361-11405.

15 C. S. Kue, S. Y. Ng, S. H. Voon, A. Kamkaew, L. Y. Chung, L. V. Kiew and H. B. Lee, Recent Strategies to Improve Boron Dipyrromethene (BODIPY) for Photodynamic Cancer Therapy: An Updated Review, Photochem. Photobiol. Sci., 2018, 17, 1691-1708.

16 L. Jean-Gérard, W. Vasseur, F. Scherninski and B. Andrioletti, Recent Advances in the Synthesis of [a]-Benzo-Fused BODIPY Fluorophores, Chem. Commun., 2018, 54, 12914-12929.

17 J. Zhao, K. Xu, W. Yang, Z. Wang and F. Zhong, The Triplet Excited State of Bodipy: Formation, Modulation and Application, Chem. Soc. Rev., 2015, 44, 8904-8939.

18 A. Kamkaew, S. Hui Lim, H. Boon Lee, L. Voon Kiew, L. Yong Chung and K. Burgess, BODIPY Dyes in Photodynamic Therapy, Chem. Soc. Rev., 2013, 42, 77-88.

19 N. Boens, V. Leen and W. Dehaen, Fluorescent Indicators Based on BODIPY, Chem. Soc. Rev., 2012, 41, 1130-1172.

20 A. Loudet and K. Burgess, BODIPY Dyes and Their Derivatives: Syntheses and Spectroscopic Properties, Chem. Rev., 2007, 107, 4891-4932.

21 J. Bañuelos, BODIPY Dye, the Most Versatile Fluorophore Ever?, Chem. Rec., 2016, 16, 335-348.

22 Z. Liu, Z. Jiang, M. Yan and X. Wang, Recent Progress of BODIPY Dyes with Aggregation-Induced Emission, Front. Chem., 2019, 7, 712 . 
23 C. Yu, Z. Huang, W. Gu, Q. Wu, E. Hao, Y. Xiao, L. Jiao and W.-Y. Wong, A Novel Family of AIE-Active Meso-2-Ketopyrrolyl BODIPYs: Bright Solid-State Red Fluorescence, Morphological Properties and Application as Viscosimeters in Live Cells, Mater. Chem. Front., 2019, 3, 1823-1832.

24 C. Duan, Y. Zhou, G.-G. Shan, Y. Chen, W. Zhao, D. Yuan, L. Zeng, X. Huang and G. Niu, Bright Solid-State Red-Emissive BODIPYs: Facile Synthesis and Their High-Contrast Mechanochromic Properties, J. Mater. Chem. C, 2019, 7, 3471-3478.

25 R. P. Paitandi, R. S. Singh, B. K. Dwivedi, V. D. Singh and D. S. Pandey, Time Dependent Aggregation Induced Emission Enhancement and the Study of Molecular Packing in Closely Related Azo-Phenol BODIPY Species, Dalton Trans., 2018, 47, 3785-3795.

26 H. Gao, Y. Gao, C. Wang, D. Hu, Z. Xie, L. Liu, B. Yang and Y. Ma, Anomalous Effect of Intramolecular Charge Transfer on the Light Emitting Properties of BODIPY, ACS Appl. Mater. Interfaces, 2018, 10, 14956-14965.

27 Q. Li and Y. Qian, Aggregation-Induced Emission Enhancement and Cell Imaging of a Novel (Carbazol-N-Yl)Triphenylamine-BODIPY, New J. Chem., 2016, 40, 7095-7101.

28 S. Zhang, Y. Wang, F. Meng, C. Dai, Y. Cheng and C. Zhu, Circularly Polarized Luminescence of AIE-Active Chiral O-BODIPYs Induced via Intramolecular Energy Transfer, Chem. Commun., 2015, 51, 9014-9017.

29 T. T. Vu, M. Dvorko, E. Y. Schmidt, J.-F. Audibert, P. Retailleau, B. A. Trofimov, R. B. Pansu, G. Clavier and R. Méallet-Renault, Understanding the Spectroscopic Properties and Aggregation Process of a New Emitting Boron Dipyrromethene (BODIPY), J. Phys. Chem. C, 2013, 117, 5373-5385.

30 S. Choi, J. Bouffard and Y. Kim, Aggregation-Induced Emission Enhancement of a Meso-Trifluoromethyl BODIPY via J-Aggregation, Chem. Sci., 2013, 5, 751-755.

31 L. Gai, H. Lu, B. Zou, G. Lai, Z. Shen and Z. Li, Synthesis and Spectroscopic Properties of Bodipy Dimers with Effective Solid-State Emission, RSC Adv., 2012, 2, 8840-8846.

32 D. Zhang, Y. Wen, Y. Xiao, G. Yu, Y. Liu and X. Qian, Bulky 4-Tritylphenylethynyl Substituted Boradiazaindacene: Pure Red Emission, Relatively Large Stokes Shift and Inhibition of Self-Quenching, Chem. Commun., 2008, 4777-4779.

33 J. Zhu, J. Zou, Z. Zhang, J. Zhang, Y. Sun, X. Dong and Q. Zhang, An NIR Triphenylamine Grafted BODIPY Derivative with High Photothermal Conversion Efficiency and Singlet Oxygen Generation for Imaging Guided Phototherapy, Mater. Chem. Front., 2019, 3, 1523-1531.

34 R. S. Singh, A. Kumar, S. Mukhopadhyay, G. Sharma, B. Koch and D. S. Pandey, An Unconventional Mechanistic Insight on Aggregation Induced Emission in Novel Boron Dipyrromethenes and Their Rational Biological Realizations, J. Phys. Chem. C, 2016, 120, 22605-22614.

35 M. H. Chua, Y. Ni, M. Garai, B. Zheng, K.-W. Huang, Q.-H. Xu, J. Xu and J. Wu, Towards Meso-Ester BODIPYs with Aggregation-Induced Emission Properties: The Effect of Substitution Positions, Chem. - Asian J., 2015, 10, 1631-1634.
36 R. S. Singh, R. K. Gupta, R. P. Paitandi, M. Dubey, G. Sharma, B. Koch and D. S. Pandey, Morphological Tuning via Structural Modulations in AIE Luminogens with the Minimum Number of Possible Variables and Their Use in Live Cell Imaging, Chem. Commun., 2015, 51, 9125-9128.

37 S. Mukherjee and P. Thilagar, Fine-Tuning Dual Emission and Aggregation-Induced Emission Switching in NPI-BODIPY Dyads, Chem. - Eur. J., 2014, 20, 9052-9062.

38 Z. Li, M. Zheng, X. Guan, Z. Xie, Y. Huang and X. Jing, Unadulterated BODIPY-Dimer Nanoparticles with High Stability and Good Biocompatibility for Cellular Imaging, Nanoscale, 2014, 6, 5662-5665.

39 R. Hu, C. F. A. Gómez-Durán, J. W. Y. Lam, J. L. BelmonteVázquez, C. Deng, S. Chen, R. Ye, E. Peña-Cabrera, Y. Zhong and K. S. Wong, et al., Synthesis, Solvatochromism, Aggregation-Induced Emission and Cell Imaging of Tetraphenylethene-Containing BODIPY Derivatives with Large Stokes Shifts, Chem. Commun., 2012, 48, 10099-10101.

40 X.-F. Zhang, BODIPY Photosensitizers Based on PET and Heavy Atom Effect: A Comparative Study on the Efficient Formation of Excited Triplet State and Singlet Oxygen in BODIPY Dimers and Monomers, J. Photochem. Photobiol., A, 2018, 355, 431-443.

41 M. T. Whited, N. M. Patel, S. T. Roberts, K. Allen, P. I. Djurovich, S. E. Bradforth and M. E. Thompson, SymmetryBreaking Intramolecular Charge Transfer in the Excited State of Meso-Linked BODIPY Dyads, Chem. Commun., 2011, 48, 284-286.

42 M. Bröring, R. Krüger, S. Link, C. Kleeberg, S. Köhler, X. Xie, B. Ventura and L. Flamigni, Bis(BF2)-2,2'-Bidipyrrins (BisBODIPYs): Highly Fluorescent BODIPY Dimers with Large Stokes Shifts, Chem. - Eur. J., 2008, 14, 2976-2983.

43 Y. Liu, N. Song, L. Chen and Z. Xie, Triple-BODIPY Organic Nanoparticles with Particular Fluorescence Emission, Dyes Pigm., 2017, 147, 241-245.

44 J. Zou, Z. Yin, K. Ding, Q. Tang, J. Li, W. Si, J. Shao, Q. Zhang, W. Huang and X. Dong, BODIPY Derivatives for Photodynamic Therapy: Influence of Configuration versus Heavy Atom Effect, ACS Appl. Mater. Interfaces, 2017, 9, 32475-32481.

45 W. Li, L. Li, H. Xiao, R. Qi, Y. Huang, Z. Xie, X. Jing and H. Zhang, Iodo-BODIPY: A Visible-Light-Driven, Highly Efficient and Photostable Metal-Free Organic Photocatalyst, RSC Adv., 2013, 3, 13417-13421.

46 K. Naim, S. T. Nair, P. Yadav, A. Shanavas and P. P. Neelakandan, Supramolecular Confinement within Chitosan Nanocomposites Enhances Singlet Oxygen Generation, ChemPlusChem, 2018, 83, 418-422.

47 K. Mochizuki, L. Shi, S. Mizukami, M. Yamanaka, M. Tanabe, W.-T. Gong, A. F. Palonpon, S. Kawano, S. Kawata and K. Kikuchi, et al., Nonlinear Fluorescence Imaging by Photoinduced Charge Separation, Jpn. J. Appl. Phys., 2015, 54, 042403.

48 Y. P. Rey, D. G. Abradelo, N. Santschi, C. A. Strassert and R. Gilmour, Quantitative Profiling of the Heavy-Atom Effect in BODIPY Dyes: Correlating Initial Rates, Atomic Numbers, 
and 1O2 Quantum Yields, Eur. J. Inorg. Chem., 2017, 2170-2178.

49 V. Lakshmi, M. R. Rao and M. Ravikanth, Halogenated Boron-Dipyrromethenes: Synthesis, Properties and Applications, Org. Biomol. Chem., 2015, 13, 2501-2517.

50 K. N. Solov'ev and E. A. Borisevich, Intramolecular HeavyAtom Effect in the Photophysics of Organic Molecules, Phys.-Usp., 2005, 48, 231.

51 S. P. McGlynn, J. Daigre and F. J. Smith, External HeavyAtom Spin-Orbital Coupling Effect. IV. Intersystem Crossing, J. Chem. Phys., 1963, 39, 675-679.

52 Z. Wang, X. Hong, S. Zong, C. Tang, Y. Cui and Q. Zheng, BODIPY-Doped Silica Nanoparticles with Reduced Dye Leakage and Enhanced Singlet Oxygen Generation, Sci. Rep., 2015, 5, 12602.

53 R. S. Llano, E. A. Zaballa, J. Bañuelos, C. F. A. G. Durán, J. L. B. Vázquez, E. P. Cabrera and I. L. Arbeloa, Tailoring the Photophysical Signatures of BODIPY Dyes: Toward Fluorescence Standards across the Visible Spectral Region, in Photochemistry and Photophysics - Fundamentals to Applications, IntechOpen, 2018, pp. 21-40.

54 A. Nagai, K. Kokado, J. Miyake and Y. Chujo, Quantum Yield and Morphology Control of BODIPY-Based Supramolecular Self-Assembly with a Chiral Polymer Inhibitor, Polym. J., 2010, 42, 37-42.

55 L. Wang, Y. Zhang and Y. Xiao, Meso-Alkoxy BODIPYs with a Good Balance between Larger Stokes Shifts and
Higher Fluorescence Quantum Yields, RSC Adv., 2013, 3, 2203-2206.

56 A. Oliden-Sánchez, R. Sola-Llano, J. Bañuelos, I. GarcíaMoreno, C. Uriel, J. C. López and A. M. Gómez, Tuning the Photonic Behavior of Symmetrical Bis-BODIPY Architectures: The Key Role of the Spacer Moiety, Front. Chem., 2019, 7, 801.

57 T. Brixner, R. Hildner, J. Köhler, C. Lambert and F. Würthner, Exciton Transport in Molecular Aggregates From Natural Antennas to Synthetic Chromophore Systems, Adv. Energy Mater., 2017, 7, 1700236.

58 J. Ahrens, A. Scheja, R. Wicht and M. Bröring, Excitonic Coupling in Acyclic and Cyclic Dithioaryl-Linked BODIPY DYEmers, Eur. J. Org. Chem., 2016, 2864-2870.

59 M. Kasha, H. R. Rawls and E.-B. M. Ashraf, The Exciton Model in Molecular Spectroscopy, Pure Appl. Chem., 1965, 11, 371-392.

60 R. Hu, E. Lager, A. Aguilar-Aguilar, J. Liu, J. W. Y. Lam, H. H. Y. Sung, I. D. Williams, Y. Zhong, K. S. Wong and E. Peña-Cabrera, et al., Twisted Intramolecular Charge Transfer and Aggregation-Induced Emission of BODIPY Derivatives, J. Phys. Chem. C, 2009, 113, 15845-15853.

61 P. Xu, Q. Qiu, X. Ye, M. Wei, W. Xi, H. Feng and Z. Qian, Halogenated Tetraphenylethene with Enhanced AggregationInduced Emission: An Anomalous Anti-Heavy-Atom Effect and Self-Reversible Mechanochromism, Chem. Commun., 2019, 55, 14938-14941. 